\title{
MALDI-TOF and FTIR microscopy analysis of blood serum from diarrhea patients
}

\author{
Mahmoud Huleihel $^{\mathrm{a}, \mathrm{b}, *}$, Mark Karpasas ${ }^{\mathrm{b}}$, Marina Talyshansky ${ }^{\mathrm{b}}$, Yelena Souprun ${ }^{\mathrm{b}}$, \\ Yelena Doubijanski ${ }^{\mathrm{b}}$ and Vitaly Erukhimovitch ${ }^{\mathrm{b}}$ \\ ${ }^{a}$ Department of Virology, Ben-Gurion University of the Negev, Beer-Sheva, Israel \\ ${ }^{\mathrm{b}}$ National Institute for Biotechnology, Ben-Gurion University of the Negev, Beer-Sheva, Israel
}

\begin{abstract}
Mass-spectroscopic and vibrational spectroscopic methods were applied for the analysis of human blood samples from healthy subjects and patients in order to detect spectral peaks which might serve as biomarkers for monitoring and identification of specific diseases. In the present study samples of serum were obtained from healthy and patient persons suffering from diarrhea and analyzed by matrix-assisted laser desorption/ionization time-of-flight (MALDI-TOF) and microscopic Fourier transform infrared spectroscopy (FTIR). The results obtained by both spectroscopic techniques show similar and consistent spectral peaks in all the examined sera obtained from healthy persons. In all tested patient samples two unique interesting peaks appeared at $m / z 11,553$ and 11,710 in MALDI-TOF spectra and a peak at area $1600 \mathrm{~cm}^{-1}$ was significantly reduced in all of the tested patient samples in FTIR spectra. These parameters might be used as a basis for developing a spectral method for the detection and identification of specific human diseases and probably other disorders. It seems that a combination of these two techniques may provide much more reliable results for the detection and identification of various abnormalities.
\end{abstract}

Keywords: MALDI-TOF, blood serum, biomarkers, FTIR-microscopy, patients, diarrhea

\section{Introduction}

Blood is known to contain a large variety of proteins characterized by different structures and functions [1,2]. Monitoring a large amount of different proteins which present in a complex mixture such as serum presents a major analytical challenge. Fortunately, the development of matrix-assisted laser desorption/ionization time-of-flight mass spectrometry (MALDI-TOF), makes it easy to detect a large number of variable proteins [3,4]. MALDI-TOF is also indispensable to elucidate the modified protein structures, which cannot be substituted by DNA technology [5]. MALDI-TOF analysis is also known to be less affected by the presence of salts compared to other forms of mass spectrometry, which makes it highly useful for direct analysis of biological samples without high levels of purifications [6,7]. Therefore, any changes in the protein comparison of the serum, could be detected easily using this technique. As it known, infection with bacteria, viruses or other pathogen or as a result of development of malignant tumor, the immune system of animals produces an immune response. The ability to monitor the immune system response of a host to an antigen is important in order to determine the type and probably the severity of the infection and possibly the identification of the antigen. In the case of cancer development, probably it will be possible to identify the kind and progress of this cancer.

\footnotetext{
* Corresponding author: Mahmoud Huleihel, The Institute for Applied Biosciences, Ben-Gurion University of the Negev, POB 653, Beer-Sheva 84105, Israel. Tel.: +972 8 6461999; Fax: +972 8 6472970; E-mail: mahmoudh@bgumail.bgu.ac.il.
} 
This technique could be used also for rapid analysis and identification of expressed protein profile in cells and tissues such as infected cells or malignant cells.

The technique generally used to monitor cellular protein expression has been 2D polyacrylamide gel electrophoresis (2D-PAGE) [8,9]. This 2D gel method, though, only provides a separation of the cell components and approximate molecular weights while exact identification must still be provided by protein sequencing or mass spectrometric methods [10-12]. In the present study we examined serum samples obtained from normal and patient persons by both MALDI-TOF and FTIR-microscopy techniques trying to detect specific biomarkers for discrimination between normal and patient persons.

FTIR spectroscopy is a noninvasive method that has been applied for identifying various biomolecular components of the cell $[13,14]$. This technique has been proved to be sensitive for discrimination between normal and cancer cells [15-18] and between normal cells and virally infected cells [19].

\section{Materials and methods}

\subsection{Sample preparation of blood for MALDI-TOF}

Blood samples were obtained from the fingers of the tested persons. Approximately $50 \mu 1$ of blood were obtained from each person. Blood samples were obtained from five healthy persons and five patient persons suffered from severe bacterial diarrhea. All tested persons were males at age between 30-45 years old. The blood samples were then centrifuged for $5 \mathrm{~min}$ at $14000 \mathrm{rpm}$ in eppendorf centrifuge and the serum was removed and separated from the cellular material. $2 \mu \mathrm{l}$ of serum was dialyzed for $30 \mathrm{~min}$ by spotting on a 0.025 MF-Millipore membrane filter (Millipore, Bedford, MA, USA) floating on distilled water. After dialysis the sample was removed and mixed with an equal amount of saturated sinapinic acid solution. Sinapinic acid (Aldrich, Milwaukee, WI, USA) was dissolved in water-acetonitrile $(1: 1)$ until saturation occurred. Samples were spotted on an autosampler plate and allowed to air dry.

\subsection{MALDI-TOF analysis}

Samples were analyzed on a PE Biosystems (Framingham, MA, USA) Voyager-Elite MALDI-TOF using $337 \mathrm{~nm}$ radiation from a nitrogen laser as described [23]. An accelerating voltage of $20 \mathrm{kV}$ was used and a two-point external calibration was performed using cytochrome $\mathrm{c}$ and sinapinic acid as the calibrants. The number of laser shots was set to 300 by steps of 30 shots. Each sample was analyzed by MALDI-TOF. The spectra were recorded in linear mode within a mass range from 2 to $20 \mathrm{kDa}$ and from 20 to $100 \mathrm{kDa}$.

\subsection{Sample preparation of blood for FTIR micoscopy}

Since ordinary glass slides exhibit strong absorption in the wavelength range of interest to us, we used zinc sellenide crystals, which are highly transparent to IR radiation. A drop of $1 \mu \mathrm{l}$ of the serum sample (of each of the 5 tested healthy persons and patients) was placed on a certain area on the zinc sellenide crystal, air dried for $30 \mathrm{~min}$ at room temperature (or for $10 \mathrm{~min}$ by air drying in a laminar flow) and examined by FTIR microscopy. 


\subsection{FTIR spectra measurement}

FTIR measurements were performed in transmission mode with a liquid-nitrogen-cooled MCT detector of the FTIR microscope (Bruker IRScope II) coupled to an FTIR spectrometer (BRUKER EQUINOX model $55 / \mathrm{S}$, OPUS software). The spectra were obtained in the wavenumber range of $600-4000 \mathrm{~cm}^{-1}$ in the mid-IR region. Spectral resolution was set at $4 \mathrm{~cm}^{-1}$ with Backman Harris 4-Term apodization. To increase the signal to noise ratio, a spectrum was taken as an average of 128 scans. Since the samples to be analyzed were often heterogeneous, appropriate regions were chosen by FTIR microscopy so as to eliminate different impurities (salts, medium residuals, etc.). The aperture used in this study was $100 \mu \mathrm{m}$, since this aperture gave the best signal/noise ratio. At lower apertures, the quality of the spectra was bad due to the high noise level. In addition, at apertures lower than $20 \mu \mathrm{m}$, there was diffraction of the IR beam. Baseline correction and normalization were obtained for all the spectra by OPUS software. Baseline correction was performed by the rubber band method as follows: each spectrum was divided up into ranges of equal size. In each range, the minimum y value was determined. The baseline was then created by connecting the minima with straight lines. Starting from "below", a rubber band stretched over this curve constituted the baseline. The baseline points that did not lie on the rubber band were discarded. Normalization was performed by a vector method, as follows. The average $y$-value of the spectrum was first calculated. This average value was then subtracted from the spectrum so that the middle of the spectrum was pulled down to $y=0$. The sum of the squares of all the $y$-values was then calculated, and the spectrum was divided by the square root of this sum. The vector norm of the resulting spectrum was 1 . Peak positions were determined by means of a second derivation method by OPUS software. For each cell type, the spectrum was taken as the average of five different measurements at various sites of the sample. Each experiment with each cell type was repeated five times. It is important to mention that there were no significant differences in the spectra from various sites (SD did not exceed 0.005).

\section{Results}

\subsection{MALDI-TOF of normal serum samples}

Serum samples obtained from healthy persons were examined by MALDI-TOF. Due to the inherent complexity of MALDI-TOF spectra generated from prefractionated clinical samples, we investigated the possibility of detecting potential biomarkers by mining the spectra using computer-based algorithms. The results presented in Fig. 1a and Fig. 2a show average spectra of the tested healthy persons. These results show consistent representative peaks all over the examined spectrum including low and high molecular weight proteins. The obtained spectra of the healthy persons can be used as a good basis for the detection of any significant change in protein composition of the serum which might be resulted from different diseases including bacterial and viral infections, cancer development, etc. One of the expected changes in the serum proteins is the production of specific proteins (antibodies) by the immune system due to infection with pathogens or cancer development. As it known the immune system responds differently to various antigens.

\subsection{MALDI-TOF of patient serum samples}

Blood samples were obtained from five different patients suffering from diarrhea. The serum of these samples was examined by MALDI-TOF and the obtained results show that the majority of the obtained spectral peaks remained unchanged both in mass value similar to the normal sera (Fig. 1b,c and 


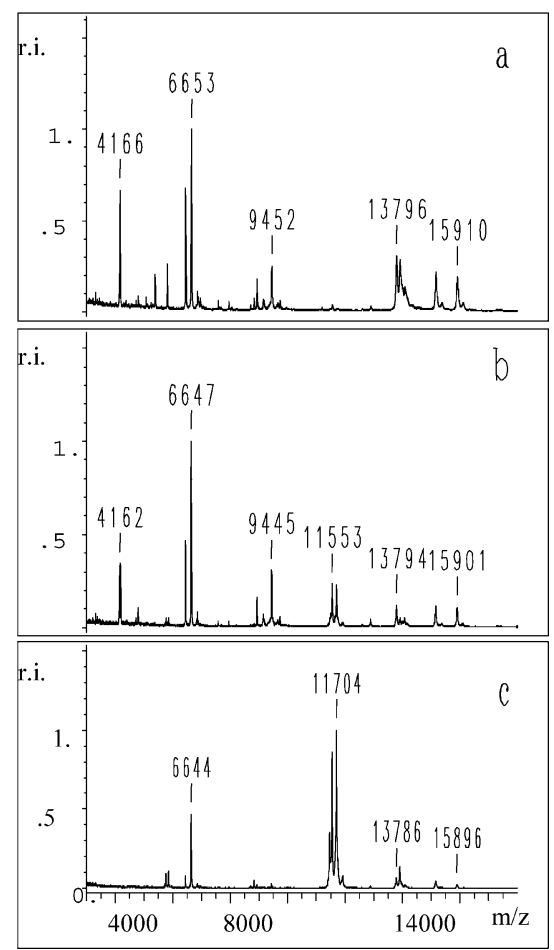

Fig. 1. MALDI-TOF spectra in the region 2000 to $\mathrm{m} / z$ 20,000 of (a) average of 5 healthy control persons, (b) average of patients 1, 2, 4 and 5 (c) patient 3.

Fig. 2b,c). However, two new peaks at $m / z 11,553$ and 11,710 appeared in the spectra of the patients which are completely missing in the serum of the normal samples. Another significant peak appeared only in one of the examined patients at $m / z 41,794$ (Fig. 2c). This peak could be related to additional abnormality rather than diarrhea. Although, other differences in peak intensities can be seen in patient samples compared to normal samples, these differences cannot be considered as useful biomarkers at the meantime due to the small number of tested patients. For deep understanding of the significance of these differences in peak intensities, further samples from similar patients should be analyzed.

\subsection{FTIR spectra of human sera from healthy and patient persons}

The serum samples obtained from healthy and patient persons were also examined by FTIR microscopy to try to find specific spectroscopic biomarkers for the detection and identification of such diseases.

Figure 3a presents the average FTIR spectra of the tested healthy subjects and patients. The spectra are dominated by the absorption bands at $1643 \mathrm{~cm}^{-1}$ and $1544 \mathrm{~cm}^{-1}$, i.e., the amide I and II bands, respectively. The amide $\mathrm{I}$ band arises from $\mathrm{C}=\mathrm{O}$ hydrogen bonded stretching vibrations, and the amide II, from $\mathrm{C}-\mathrm{N}$ stretching and $\mathrm{CNH}$ bending vibrations [20]. It can be seen clearly that a significant peak at area position $1600 \mathrm{~cm}^{-1}$, between the peaks of amide I and II, almost completely disappeared in all of the tested patient samples (Fig. 3b). This peak, for instance, may be used as biodetectors for specific abnormality.

Among the various mathematical methods applied for classification in biology and medicine cluster analysis is one of the simplest and most rapid procedures [21,22]. In our study, this technique was used 


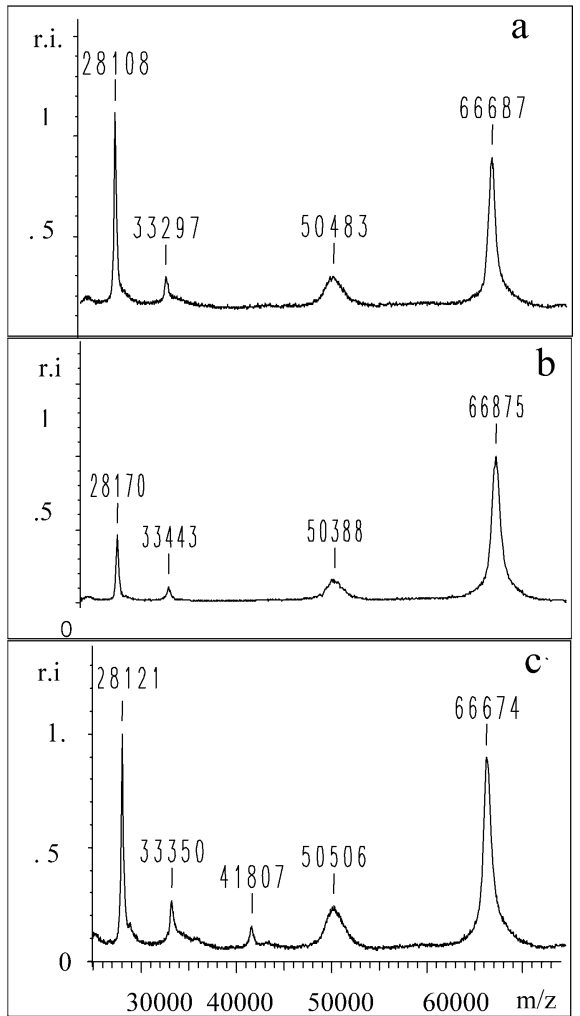

Fig. 2. MALDI-TOF spectra in the region 20,000 to $\mathrm{m} / z$ 100,000 of (a) average of 5 healthy control persons, (b) average of patients 1, 2, 4 and 5 (c) patient 3 .

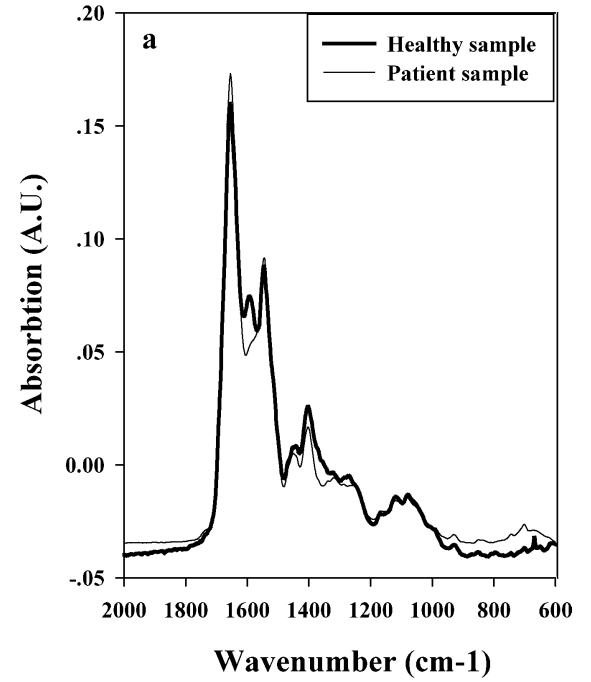

(a)

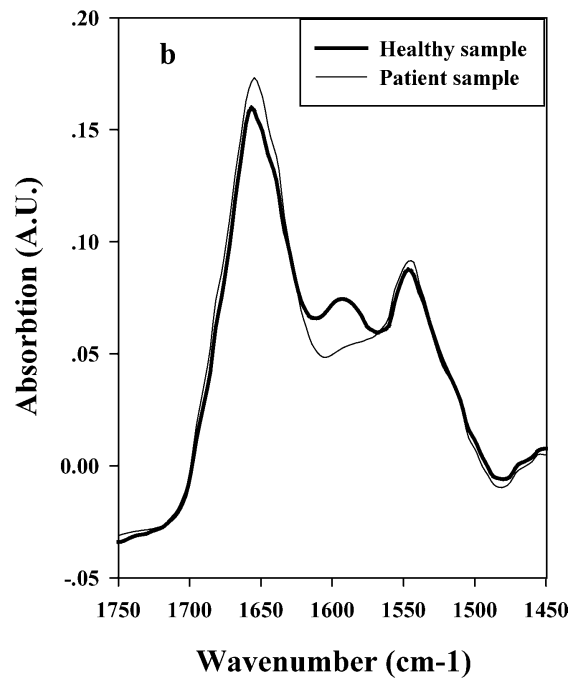

(b)

Fig. 3. (a) FTIR spectra in the region of $600-2,000 \mathrm{~cm}^{-1}$ of healthy and patient persons suffered from diarrhea. (b) FTIR spectra in the region of $1450-1750 \mathrm{~cm}^{-1}$ of healthy and patient persons suffered from diarrhea. The presented spectra are an average of 5 patient samples and 5 healthy person samples. 


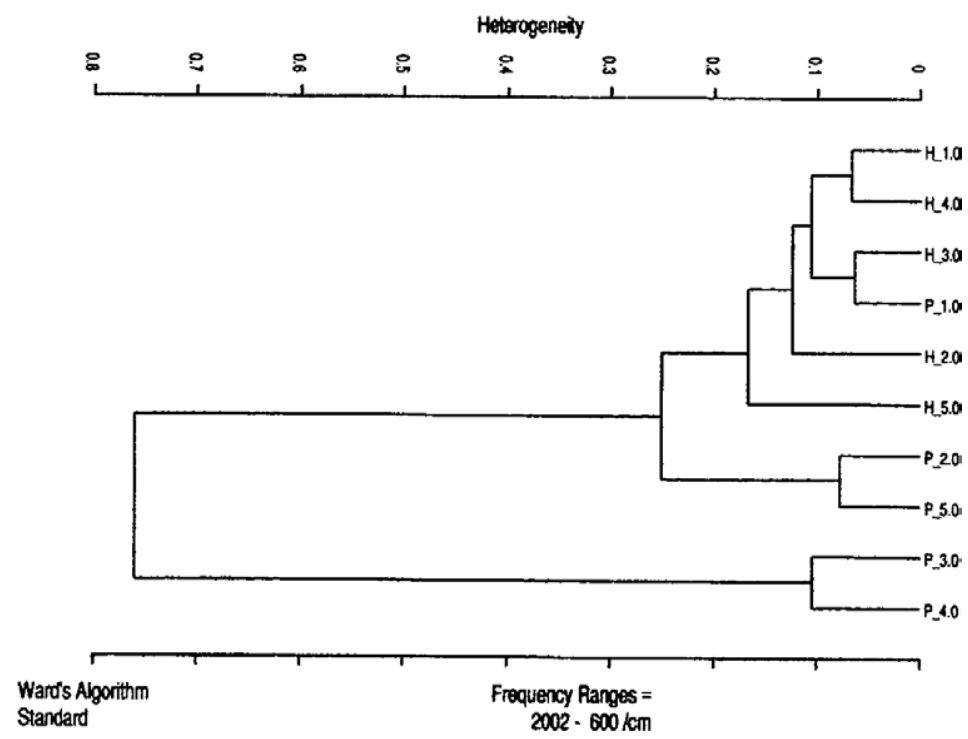

Fig. 4. Cluster analysis of the IR spectra of the examined healthy and patient samples in the frequency range $600-2000 \mathrm{~cm}^{-1}$. H-represents healthy samples and P-represents patient samples.

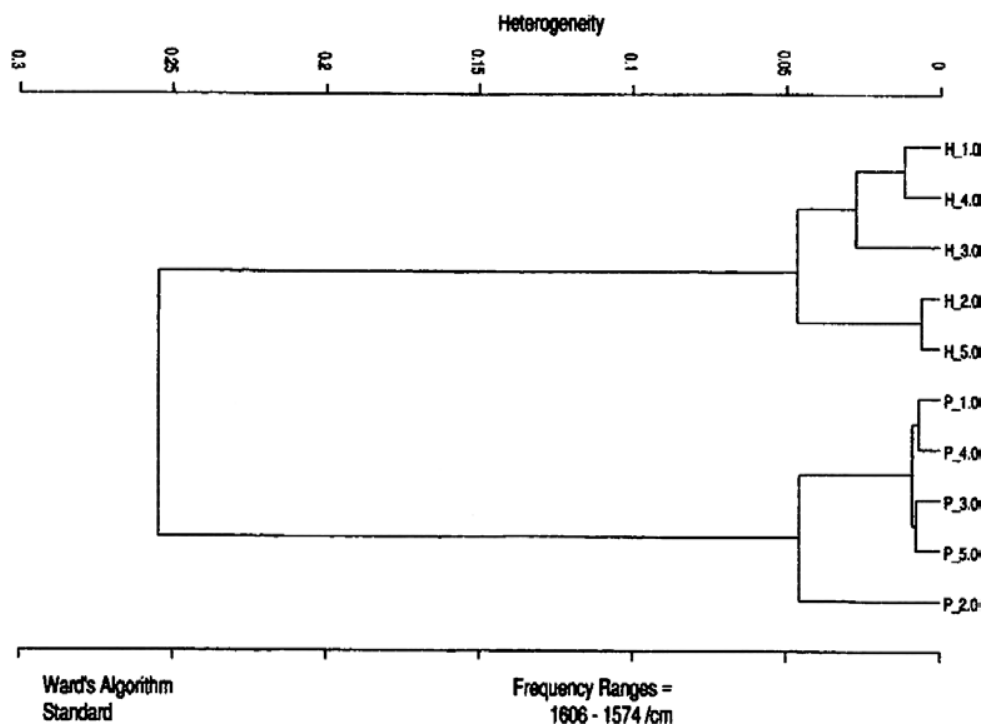

Fig. 5. Cluster analysis of the IR spectra of the examined healthy and patient samples in the frequency range $1574-1606 \mathrm{~cm}^{-1}$. H-represents healthy samples and P-represents patient samples.

to classify certain regions of the FTIR spectra of the examined healthy and patient samples. Inspection of the entire spectrum from $600-2000 \mathrm{~cm}^{-1}$ failed to distinguish between healthy and patient samples (Fig. 4). However, excellent classification was obtained for limited regions, such as the $1574-1606 \mathrm{~cm}^{-1}$ range (Fig. 5). 


\section{Discussion}

In the present study we examined the potential of two different easy and rapid techniques for detecting and identifying human abnormalities. Developing specific biomarkers for the detection and identification of human abnormality both by MALDI-TOF and FTIR microscopy could be highly important for future rapid and reliable detection and identification of these abnormalities. Quick and reliable identification of the disease might be critical, in many cases, for effective treatment.

The results obtained by either of these techniques are extremely promising and interesting. In MALDI-TOF two new peaks at $m / z 11,553$ and 11,710 appeared in the spectra of all the examined patients which are completely missing in the serum of the normal samples (Fig. 1b,c). These peaks could be related to the immune response.

It is interesting that such peaks appeared at $m / z 11,553$ and 11,710 were observed previously in sera of mice which were infected with two different bacteria [23]. These peaks also were observed in immunodeficient nu/nu "nude" mice infected with the same bacteria [23]. Nude mice are well known for being immunodeficient owing to the absence of a thymus which is necessary for normal T-cell function [24,25]. So, these peaks might be considered as a response of the immune system of the host, although the results obtained with the "nude" mice are not exactly fit with this possibility.

Certainly examination of further samples of patients with different diseases caused by viral or bacterial infections are necessary for deep understanding of the meaning and the significance of these peaks and possibly other spectral peaks.

On the hand, these MALDI-TOF results are supported by our FTIR examinations which showed clear and significant reduction of a peak at area $1600 \mathrm{~cm}^{-1}$ in patient samples compared to examined healthy ones (Fig. 3b). A peak in the region $1560-1630 \mathrm{~cm}^{-1}$ from blood plasma has been reported previously and assigned as $\left(\mathrm{NH}_{2}\right)$ from amino acids [26]. It is interesting to mention that this peak was observed to increase with exercise. The observed changes in this peak either in our or Deleris [26] studies might be related to the metabolic changes accompanied with human physical condition.

Although the results obtained in this study could be considered only as preliminary results, but still the interesting different consistent spectral behavior of the patients compared to the healthy samples observed by both techniques maybe considered as a promising basis for a future study including large number of samples from different patients with a variety of causes. In addition, it seems to be that a combination between these two techniques may provide much more reliable results for the detection and identification of various abnormalities. Furthermore, for each technique only a small amount of serum (1-2 $\mu \mathrm{l})$ is required, which can be easily obtained from any patient, and the final results could be obtained during very short time (approximately $1 \mathrm{hr}$ ). It is, therefore, worthwhile to continue developing each of these techniques as an efficient and reliable tool for the diagnosis and identification of human diseases and may be other abnormalities.

\section{References}

[1] J.W. Keyser, ed., Human Plasma Proteins, Wiley, New York, 1979.

[2] F. Haurowitz, The Chemistry and Function of Proteins, 2nd edn, Academic Press, New York, 1963.

[3] M. Karas and F. Hillenkamp, Anal. Chem. 60 (1988), 2299-2301.

[4] R. Kratzer, C. Eckerskorn, M. Karas and F. Lottspeich, Electophoresis 19 (1998), 1910-1919.

[5] A. Shimizu, T. Nakanishi, M. Kishikawa and A. Miyazaki, J. of Chromatography B 776 (2002), 15-30.

[6] M. Mann and G. Talbo, Curr. Opin. Biotechnol. 7 (1996), 11-19.

[7] R. Wang and B.T. Chait, Curr. Opin. Biotechnol. 5 (1994), 77-84. 
[8] P.H. O'Farrell, J. Biochem. 250 (1975), 4007-4021.

[9] J.R. Strahler, R. Kuick and S.M. Hanash, in: Protein Structure. A Practical Approach, T. Creighton, ed., IRL Press, Oxford, England, 1989, pp. 231-245.

[10] X. Liang, J. Bai, H.Y. Liu and D.M. Lubman, Anal. Chem. 19 (1996), 1012-1018.

[11] A. Shevchenko, M. Wilm, O. Vorm and M. Mann, Anal. Chem. 68 (1996), 850-858.

[12] P. Courchesne, R. Luethy and S.D. Patterson, Electrophoresis 18 (1997), 369-381.

[13] H.H. Mantsch and D. Chapman, Infrared Spectroscopy of Biomolecules, Wiley, N.Y., 1996.

[14] M. Jackson, K. Kim, J. Tetteh, J.R. Manfield, B. Dolenko, R.L. Somorjai, F.W. Orr, P.H. Watson and H.H. Mantsch, SPIE 3257 (1998), 24-34.

[15] E. Gazi, J. Ddwyer, P. Gardner, A. Ghanbari-Siahkali, A.P. Wade, J. Miyan, N.P. Lockyer, J.C. Vickerman, M.W. Clarke, J.H. Chanks, L.J. Scott, C.A. Hart and M. Brown, J. Pathol. 201 (2003), 99-108.

[16] M. Diem, L. Chiriboga, P. Lasch and A. Pacifico, Biopolymers 64 (2002), 349-353.

[17] M. Huleihel, V. Erukhimovitch, M. Talyshinsky and M. Karpasas, Appl. Spectroscopy 56 (2002), 640-645.

[18] V. Erukhimovitch, M. Talyshinsky, Y. Souprun and M. Huleihel, Photochem. Photobiol. 76 (2002), 446-451.

[19] A. Salman, V. Erukhimovitch, M. Talyshinsky, M. Huleihil and M. Huleihel, Biopolymers 67 (2002), 406-412.

[20] P. Lasch and D. Naumann, Cell. Mol. Biol. 44 (1998), 189-201.

[21] C. Kristensen, N. Ashkenasy, R. Jain and A. Koretsky, Br. J. Cancer 79 (1999), 278-285.

[22] D.M. Haaland, H.D.T. Jones and E.V. Thomas, Appl. Spectros. 51 (1997), 340-345.

[23] A.M. Haag, J. Chaiban, K.H. Johnston and B.C. Richard, J. Mass Spectrom. 36 (2001), 15-20.

[24] E.M. Pantelouris, Nature (London) 217 (1968), 370-371.

[25] C.T. Hansen, in: The Nude Mouse in Experimental and Clinical Research, F. Fogh and B.C. Giovanella, eds, Academic Press, New York, 1978, pp. 1-15.

[26] G. Déléris and C. Petibois, Vib. Spec. 32 (2003), 129-136. 


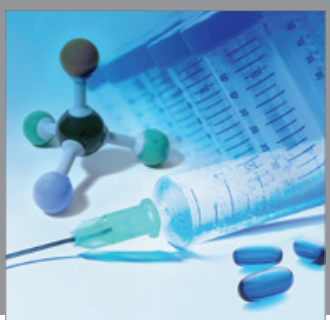

International Journal of

Medicinal Chemistry

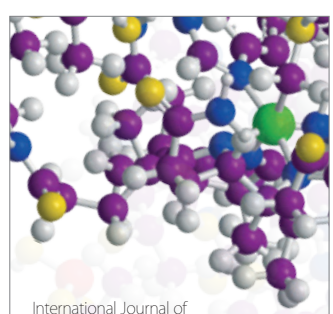

Carbohydrate Chemistry

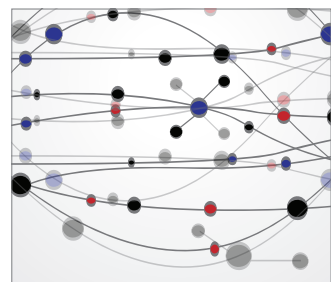

The Scientific World Journal
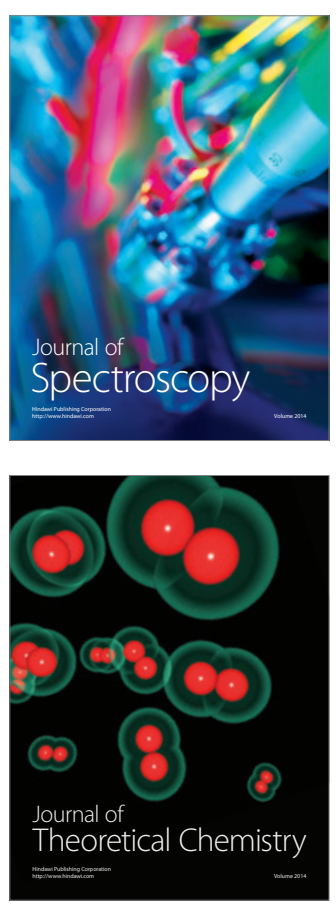
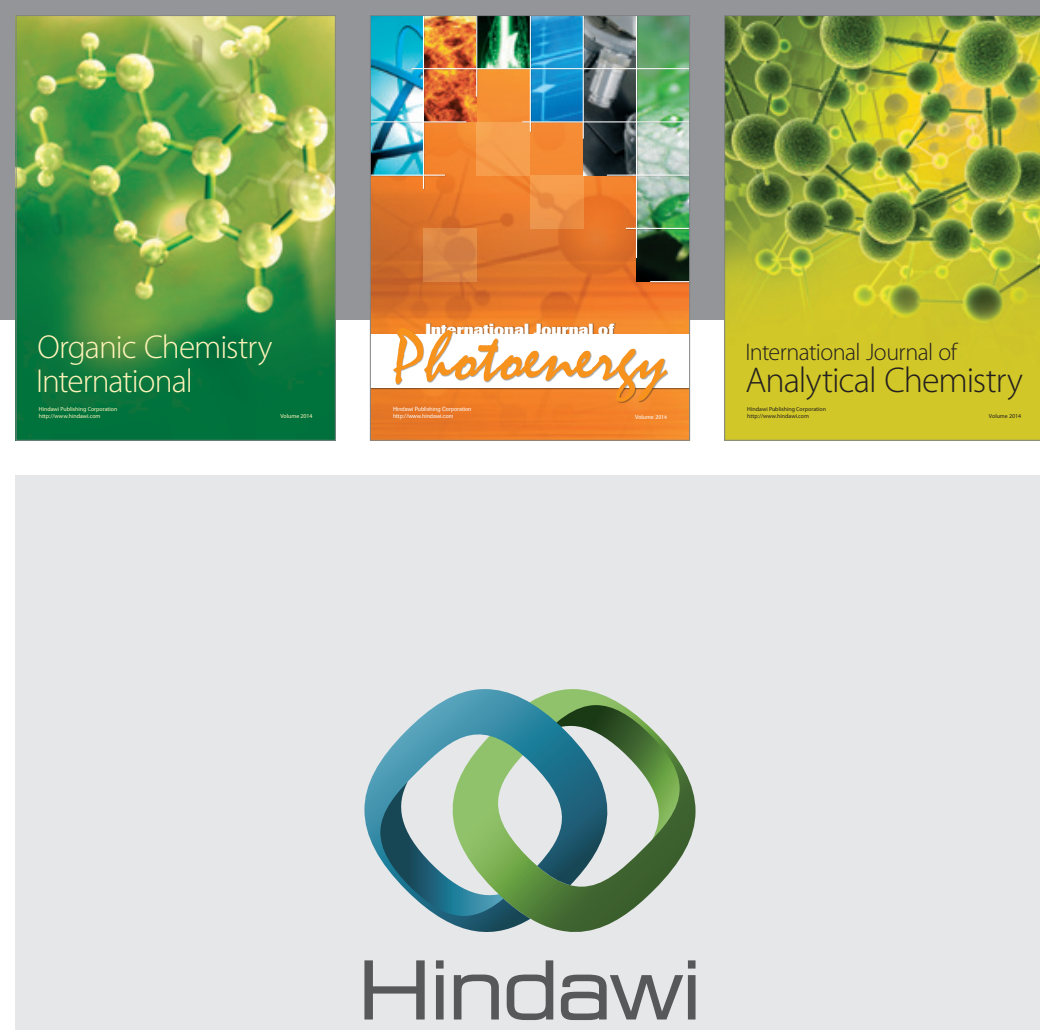

Submit your manuscripts at

http://www.hindawi.com
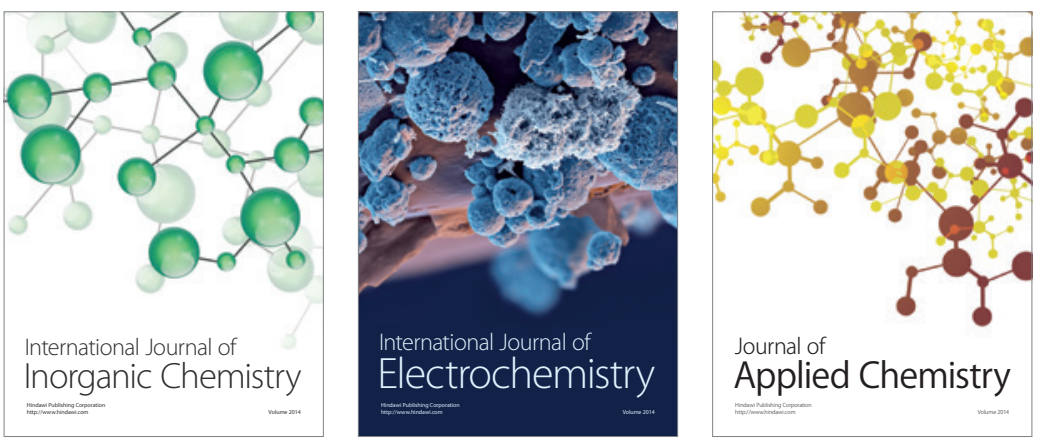

Journal of

Applied Chemistry
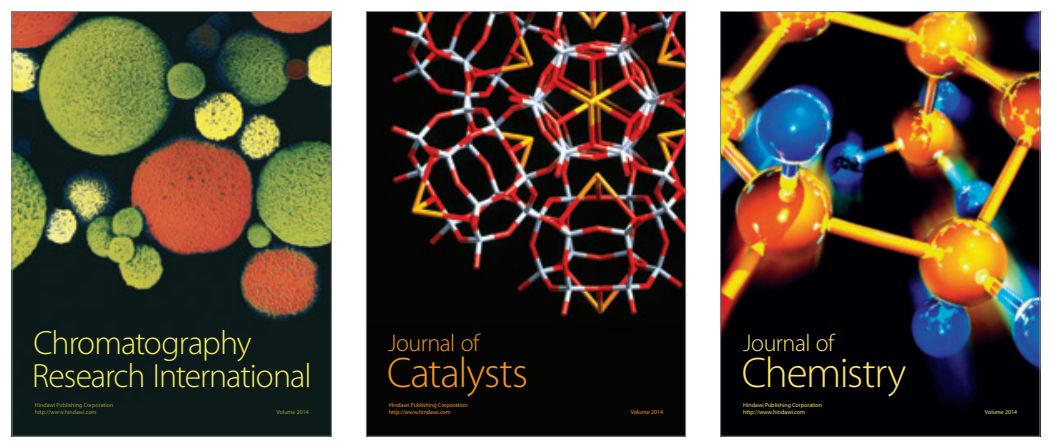
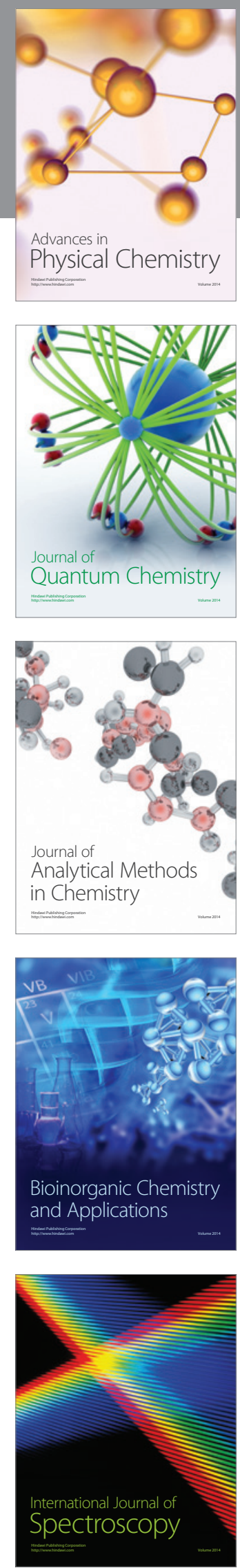